\title{
Daptomycin: a novel lipopeptide antibiotic against Gram-positive pathogens
}

This article was published in the following Dove Press journal:

Infection and Drug Resistance

27 August 2010

Number of times this article has been viewed

\author{
Andres Beiras-Fernandez ${ }^{1, *}$ \\ Ferdinand Vogt 1 ,* \\ Ralf Sodian' \\ Florian Weis ${ }^{2}$ \\ 'Department of Cardiac Surgery, \\ University Hospital Großhadern, \\ Ludwig-Maximilian-University, \\ Munich, Germany; ${ }^{2}$ Department of \\ Anesthesiology, University Hospital \\ Großhadern, Ludwig-Maximilian- \\ University, Munich, Germany \\ *Andres Beiras-Fernandez and \\ Ferdinand Vogt contributed equally to \\ this paper
}

\begin{abstract}
The aim of this review is to summarize the historical background of drug resistance of Gram-positive pathogens as well as to describe in detail the novel lipopeptide antibiotic daptomycin. Pharmacological and pharmacokinetic aspects are reviewed and the current clinical use of daptomycin is presented. Daptomycin seems to be a reliable drug in the treatment of complicated skin and skin structure infections, infective right-sided endocarditis, and bacteremia caused by Gram-positive agents. Its unique mechanism of action and its low resistance profile, together with its rapid bactericidal action make it a favorable alternative to vancomycin in multi-drug resistant cocci. The role of daptomycin in the treatment of prosthetic material infections, osteomyelitis, and urogenital infections needs to be evaluated in randomized clinical trials.
\end{abstract}

Keywords: daptomycin, multi-drug resistance, methicillin-resistant Staphylococcus aureus (MRSA), pneumonia, urinary tract infection, left-sided endocarditis

\section{Introduction}

Gram-positive pathogens have been a challenge to physicians throughout the history of medicine. Multi-drug resistance is an emerging threat in the treatment of in- and outpatient infections caused by staphylococci, streptococci and enterococci. ${ }^{1}$ Growing interest in safe and effective antibiotics for the treatment of infections related to resistant Gram-positive bacteria has led to the development and marketing of new substances in recent years, eg, linezolid, tigecyclin, quinupristin-dalfopristin, retapamulin, and daptomycin. This last drug, a lipopeptide antibiotic, has already been successfully used in the on- and off-label treatment of severe Gram-positive bacterial infections including multi-drug resistant pathogens. Daptomycin, the first-in-class cyclic lipopeptide antibiotic, was approved in Europe for the treatment of complicated skin and soft tissue infections in 2006 and for the treatment of right-sided infective endocarditis due to Staphylococcus aureus and S. aureus bacteremia in $2007 .{ }^{2}$

The aim of this review is to summarize daptomycin's unique pharmacological and pharmacokinetic properties, its clinical application, and its significance for the treatment of multi-drug resistant pathogens. Detailed information about experimental data on microbiological and pharmacological aspects is beyond the scope of this review, but may be easily found elsewhere. ${ }^{3}$

\section{History of drugs and drug-resistance in the treatment of Gram-positive microbes}

The clinical discovery and early development of penicillin (1928-1940) was a breakthrough in the treatment of a number of serious infections (ie, complicated skin and 
skin-structure infections (cSSSIs), pneumonia, and others) ${ }^{4}$ The first penicillin-resistant cocci were found already in 1944 and the mechanism of penicillinases was detected. ${ }^{5}$ In 1956 methicillin was introduced into clinical practice and only two years later the first methicillin-resistant strain of $S$. aureus (MRSA) had been isolated. ${ }^{6}$ Since then, the prevalence of MRSA and methicillin-resistant Staphylococcus epidermidis has steadily increased. In a recent study from the US including 422 emergency department patients, $59 \%$ of $S$. aureus isolates from skin and soft tissue infections were resistant to methicillin, with variations from $20 \%$ to $72 \%{ }^{7}$

The prevalence of community acquired-MRSA is not uniform in Europe. It ranges from low in France $3.6 \%$ of MRSA) and in England, and Wales ${ }^{8,9}$ to high in Greece, with $75 \%$ of MRSA strains in the community. ${ }^{10}$ The prevalence is also high in Algeria, with $72 \%$ of the isolates being MRSA. ${ }^{11}$ Whereas in 1995 less than $40 \%$ of all infections in North-American intensive care units (ICU) were due to MRSA, it was more than $60 \%$ in $2004 .{ }^{12}$ In the same year $22.6 \%$ of all isolates of $S$. aureus in Germany were MRSA.

Vancomycin, a tricyclic glycopeptide produced by Streptomyces orientalis, was discovered in 1956 and has been the drug of choice for decades in the treatment of patients with MRSA infections. ${ }^{13}$ Vancomycin is an antibiotic with a number of limitations, beginning with limited levels in many kinds of tissue and numerous side-effects, like nephro- and ototoxicity. Rapid infusion ( $<10 \mathrm{~min}$ ) may lead to the "red-man-syndrome", by liberation of huge amounts of histamine. ${ }^{14}$ Furthermore, Von Drygalski et al found vancomycin to be able to cause immune thrombocytopenia by induction of auto-antibodies. ${ }^{15}$ Correct dosage of vancomycin is difficult and many authors recommend drug monitoring. Recent data seem to show that continuous infusion of vancomycin may be the best mode of application in patients suffering from acute renal failure, requiring hemofiltration in an ICU setting. ${ }^{16-18}$ The minimum inhibitory concentration of vancomycin has risen in the last decade and consequently the susceptibility break points have been lowered. ${ }^{19}$ In 1996 the first vancomycin (glycopeptides) intermediate susceptible $S$. aureus (VISA) (interchangeably GISA) was described and in the first vancomycin-resistant S. aureus (VRSA) was detected. ${ }^{20}$ Since the use of vancomycin has steadily increased in the following decades, ${ }^{21}$ the necessity towards newer antibiotics with bactericidal effects upon these pathogens and a more favorable profile of side effects has grown.
Linezolid is the first marketed antibiotic of the oxazolidinone class with demonstrated activity against antibiotic-susceptible and antibiotic-resistant aerobic Gram-positive cocci, including MRSA, methicillin-resistant coagulase-negative staphylococci, VRSA, and vancomycin-resistant enterococci. Development of linezolid was an important addition to the armamentarium of infectious disease specialists in response to the increasing prevalence of multiple drug-resistant (MDR) Gram-positive pathogens. The possibility of oral administration is a further advantage of linezolid. However, some known side effects, eg, bone marrow depression, limit the use of linezolid to high-risk patients, eg, after solid organ transplantation.

In this context, the in vitro data on drug resistance for daptomycin are promising. Silverman et al found a spontaneous rate of resistance for $S$. aureus of $<10^{-10}$, and for $S$. epidermidis, Enterococcus faecalis, Enterococcus faecium and Streptococcus pneumoniae of $<10^{-9} .^{22}$ Since its use in the clinical practice in 2003, more than 120,000 patients have received daptomycin and there have been about 13 cases documented in which resistances could be identified. ${ }^{23}$ In cases of reduced susceptibility, combination with other antimicrobial drugs (ie, gentamycin or rifampicin) may be advantageous. ${ }^{24}$ In most of these the focus of infection could not be eradicated.

\section{Chemical and historical aspects of daptomycin}

Daptomycin (LY 146032) is a cyclic lipopeptide, derived from a fermentation product of Streptomyces roseoporus. It consists of a 13-member amino acid cyclic lipopeptide (hydrophilic core) with a decanoyl side chain (lipophilic tail).

The drug was first discovered in the 1980s. The initial trials dealt with a dose of $4 \mathrm{mg}$ twice daily and had to be stopped in 1991 because of frequent elevations of serum levels of creatine kinase (CK) probably related to skeletal muscle toxicity. ${ }^{25}$ In 1999 the drug was re-introduced into clinical trials. The FDA approved daptomycin for the treatment of cSSSIs at a dose of $4 \mathrm{mg} / \mathrm{kg}$ daily (preceded by a starting dose of $6 \mathrm{mg} / \mathrm{kg}$ / day) at last in $2003 .^{26,27}$ In 2006 an additional FDA approval was granted for the treatment of bloodstream infections and right-sided endocarditis caused by methicillin-sensitive S. aureus (MSSA) and MRSA. The approval was confirmed in Europe in 2007.

\section{Antibacterial properties and pharmacological profile of daptomycin}

The bactericidal mechanism of daptomycin may be divided into two parts. First of all, the lipophilic decanoyl side 
chain is irreversibly bound and then calcium-dependently inserted into the bacterial cell wall without entering the cell's cytoplasm. ${ }^{28}$ This binding leads to a second reaction consisting of a disruption of the cell membrane, followed by a rapid potassium efflux with subsequent loss of the ion concentration gradient, depolarization of the cell membrane, and a subsequent disruption of the production of macromolecules like RNA, DNA and other proteins. ${ }^{29,30}$ Daptomycin exerts its bactericidal action in a rapid ( $<60 \mathrm{~min})$ and concentration dependent way. The area under the concentration-time curve is $747 \mu \mathrm{g} / \mathrm{h} / \mathrm{mL}$ at $6 \mathrm{mg} / \mathrm{kg}$ and $494 \mu \mathrm{g} / \mathrm{h} / \mathrm{mL}$ at $4 \mathrm{mg} / \mathrm{kg}$ dosage IV. The maximal plasma concentration (Cmax) is $98.9 \mu \mathrm{g} / \mathrm{mL}$ and $57.8 \mu \mathrm{g} / \mathrm{mL}$, respectively. ${ }^{31}$ A post-antibiotic effect, as known for fluoroquinolones, has also been described for daptomycin: dependent on the calcium concentration, this effect may last for 0.5 to 6.8 hours in staphylococci, enterococci and streptococci, as documented in the literature. ${ }^{32,33}$ The serum half-life of daptomycin is 8.6 hours at $4 \mathrm{mg} / \mathrm{kg} /$ day and 8.1 hours at $6 \mathrm{mg} / \mathrm{kg} /$ day. It is highly protein-bound $(91 \%-96 \%)$ which in combination with its inability to cross cell membranes results in a low volume of distribution $(0.091 / \mathrm{kg}){ }^{34,35}$ Daptomycin is a large molecule and therefore cannot pass through the gastrointestinal mucosa. Thus, the enteral application is not available. This property of daptomycin is a hindrance in terms of clinical application. However, this property may limit the non-controlled use of daptomycin in the outpatient setting, reducing therefore the risks of developing resistance. Daptomycin is excreted mainly unchanged in the urine (60\%) and therefore the dosing interval has to be increased to 48 hours in patients with a creatinine clearance below $30 \mathrm{~mL} / \mathrm{min}^{36}$ Daptomycin also shows efficacy against Gram-positive pathogens, including multi-drug resistant staphylococci, enterococci, and even VISA and vancomycin-resistant enterococcus (VRE) in vitro.

\section{Side effects and safety profile of daptomycin}

Safety aspects and tolerability have been documented in a number of trials. However, many of the observed potential side effects (AEs) were not clearly attributable to the study medication. The most frequently experienced AEs were gastrointestinal disturbances (ie, constipation, nausea and vomiting and diarrhea), reactions at the injection sites and headache. All these effects were observed in frequencies similar to the comparator drugs (ie, 3\%-6\%). Musculoskeletal side effects (ie, rise in CK) were the cause for termination of the initial studies on daptomycin in 1985. Interestingly, this effect is probably due to micro- scopic degenerations and regenerations of muscle fibers. However, as this process does not result in cell lysis and is firstly related to the dosing interval, but not to the plasma levels of daptomycin or drug accumulation, ${ }^{37}$ it does not seem to be relevant. Of the patients in the daptomycin group of the trial by Arbeit et al, 2.8\% showed elevated levels of $\mathrm{CK}$, all returning to normal within two weeks. ${ }^{38}$ Although recent reports emphasize the clinical safety of daptomycin, even with doses of more than $6 \mathrm{mg} / \mathrm{kg} / \mathrm{day},{ }^{39}$ severe rhabdomyolisis may even occur with current dosing regimens. ${ }^{40}$ In the endocarditis/bacteremia trial, 6.7\% of the patients in the daptomycin group showed elevated levels of $\mathrm{CK}$, which were significantly higher than in the comparator group $(P=0.04){ }^{41}$

In the same trial, $9.2 \%$ of the patients who received daptomycin developed peripheral neurological symptoms (ie, paresthesias and dysesthesias) which were significantly more common than in the comparator group (1.7\%). However, all symptoms were classified as mild to moderate in severity and most were short-lived and completely reversible despite continued treatment.

\section{Clinical applications of daptomycin Daptomycin for CSSSls}

Daptomycin received FDA approval for CSSSIs in 2003 on the basis of two randomized evaluator-blinded phase III studies, comparing daptomycin ( $4 \mathrm{mg} / \mathrm{kg} /$ day) either to vancomycin $(1 \mathrm{~g} / 12 \mathrm{~h})$ or an oxacillin class antibiotic (flucloxacillin, cloxacillin, oxacillin or nafcillin at a dose of 4 to $12 \mathrm{mg} / \mathrm{kg}$ /day). ${ }^{27,42,43}$ Some interesting details may be mentioned: In both groups (daptomycin and comparator group) about $10 \%(10 \%$ and $9.3 \%)$ had infections due to MRSA, with $S$. aureus being the leading cause of infection in the whole study population. Daptomycin was shown to be safe (no higher incidence of side effects) and effective with respect to the kind of infecting agents and the site of infection. Furthermore, retrospective data reported in the CORE study support the effectiveness and safety of daptomycin in the treatment of cSSSI. ${ }^{44}$

\section{Daptomycin for right-sided endocarditis and bacteremia}

In 2006 Fowler et al published a randomized controlled trial which compared daptomycin to vancomycin or antistaphylococcal penicillins in combination with gentamycin. ${ }^{45}$ On the whole, daptomycin and standard treatment were equally effective at the end of treatment (61\% vs $60.9 \%$ ) and 42 days after the end of treatment (44.2\% vs $41.7 \%$ ). 


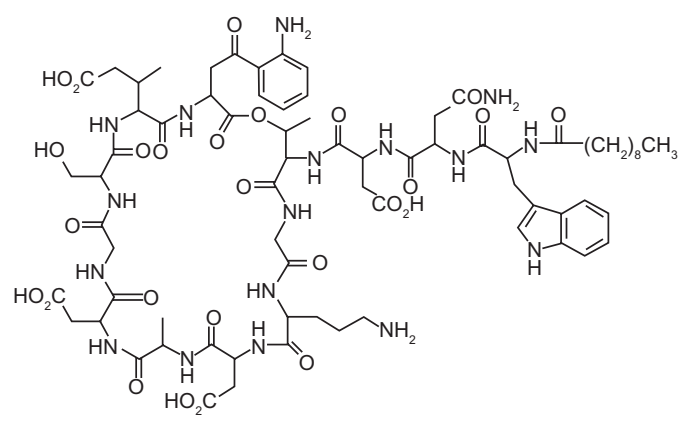

Figure I Chemical structure of daptomycin.

Some limitations of the trial may be mentioned. First of all, there were more relapsing infections due to $S$. aureus in the daptomycin group ( $15.8 \%$ vs $9.6 \%$; not statistically significant). Furthermore, the trial cannot give significant information about left-sided endocarditis, as only 18 patients with this disease were included. The success rate in this group was very low in both study groups. In conclusion, daptomycin seems to be a promising alternative to standard therapeutic approaches in patients with right-sided endocarditis and bacteremia.

\section{Off-label use of daptomycin}

\section{Bone and joint infections}

Daptomycin was found to be a promising option in the therapy of patients presenting with bone and joint infections by Falagas et al. They resumed the clinical use of this lipopeptide antibiotic in patients with bone and joint infections in a systematic review. ${ }^{46}$ In different case reports, including discitis, osteomyelitis, hip and knee infection, septic arthritis, as well as different joint inflammatory processes, daptomycin proved efficient with an initial full remission of the infection of $58 \%$. The decision to switch to daptomycin was taken after failure of the administered monotherapy with vancomycin alone, or in combination with other antibiotics. MRSA was the predominant pathogen $(83 \%)$ in the cases presented. Some case series $^{47-49}$ documented the safe use of daptomycin in patients $(n=53)$ suffering from bone and joint infections, the principal indication being osteomyelitis. In these patients, MRSA was again the predominant responsible pathogen (73\%) and the reported cure of infection after intravenous administration of a standard dose of 4-6 mg/kg/day was $81 \%$. Although the effective use of daptomycin in joint and bone infections documented by Falagas et $\mathrm{al}^{50}$ is almost exclusively in orthopedic patients, this report must be kept in mind for decision-making in heart surgery patients presenting with multi-resistant Grampositive infections of the sternotomy wound, which commonly originate osteomyelitis and presents almost no other surgical alternative..$^{51,52}$

The successful treatment of prosthetic material with daptomycin has also been documented in the literature. Antony et al and Carlyn et al reported the use of daptomycin in knee prostheses MRSA infection ${ }^{47,53}$ Furthermore, Cunha et al employed daptomycin successfully in the treatment of different prosthetic material infections, including MRSA infection of a pacemaker ${ }^{54}$ and a MSSA infection of a peripherally inserted central catheter. ${ }^{55}$ Weis et al reported the treatment with daptomycin of a patient presenting with a MRSA infection in a biventricular assist device. ${ }^{56}$ Presterl et al reported recently the use of daptomycin in cardiac device infections with $S$. epidermidis. ${ }^{57}$ Beiras-Fernandez et al documented a case series of 8 patients presenting with ventricular assisted devices successfully treated with daptomycin after multidrug resistant pathogen infection.

\section{Urinary tract infections}

Daptomycin presents favorable pharmacokinetics for this kind of infection as $66 \%$ of the active drug is excreted in the urine.$^{58}$ Furthermore, in vitro data show a good activity of daptomycin in comparison to linezolid and vancomycin for Gram-positive uropathogens causing complicated urinary tract infections. However, these promising results have not yet been confirmed by clinical trials. ${ }^{59}$

\section{Community-acquired pneumonia (CAP)}

Daptomycin was ineffective for CAP treatment in two phase III non-inferiority trials (cure rate $79 \%$ vs $87 \%)^{27}$ when compared to ceftriaxone. A murine in vitro model demonstrated that the pulmonary surfactant acts as a scavenger for daptomycin, leading to high levels in the epithelial lining fluid, but a low insertion rate into the bacterial cell wall. ${ }^{60}$ Daptomycin inserts preferentially into pulmonary surfactant and thus shows a significant elevation of the MIC in lungs with rising concentrations of surfactant. In patients with hematogeneous pneumonia the results might be better.

\section{Left-sided endocarditis}

Daptomycin has been approved for use in bacteremia and rightsided endocarditis in 2007; however, left-sided endocarditis therapy remains off-label. Different experimental models involving different pathogens such as MRSA, MSSA, Enterococcus spp, and VRE for left-sided endocarditis have shown 
a very promising antimicrobial activity of daptomycin ${ }^{61-64}$ Furthermore, Cunha et al and Mergenhagen et al reported successful treatment with $6 \mathrm{mg} / \mathrm{kg} /$ day daptomycin of MRSA left-sided endocarditis. ${ }^{65}$ Falagas et al performed a systematic review of the use of daptomycin in patients presenting with endocarditis and included some cases regarding its successful use in left-sided endocarditis. ${ }^{66}$

Although daptomycin proved efficient in some of the above stated indications, further clinical evidence in form of randomized clinical trials is needed to evaluate its effectiveness and safety in osteomyelitis, mediastinitis, joint infection, prosthetic material infections and left-sided endocarditis.

\section{Experimental data on topical application}

Despite the very modest extent of side-effects described for daptomycin, experimental use of topical daptomycin has been brought forward in recent years. ${ }^{67}$ Antibiotic-loaded bone-cement seems to be the most promising option in this field. It seems to be possible to provide sufficient peak and sustained concentrations of daptomycin to inhibit bacterial growth even in infected bones. ${ }^{68}$ The clinical relevance of these data will have to be proven by randomized controlled trials.

\section{Conclusions}

Development of new antibiotics is essential due to the increasing incidence of antibiotic drug resistance. Daptomycin, a new lipopeptide antibiotic, has proven a successful and less toxic alternative to vancomycin in the treatment of multidrug resistant Gram-positive pathogens. It seems to be a reliable drug in the treatment of cSSSIs, infective right-sided endocarditis, and bacteremia caused by Gram-positive agents. The role of daptomycin in the treatment of prosthetic material infections, mediastinitis, osteomyelitis, and urogenital infections needs to be further evaluated.

\section{Disclosure}

Andres Beiras-Fernandez has received research grants from Novartis for infection projects/studies, and is a member of a Novartis Speakers' Panel.

\section{References}

1. Enoch DA, Bygott JM, Daly ML, Karas JA. Daptomycin. J Infect. 2007;55:205-213.

2. Novartis Europharm Ltd. Summary of Product Characteristics. 2009.

3. Kanafani ZA, Corey GR. Daptomycin: a rapidly bactericidal lipopeptide for the treatment of Gram-positive infections. Expert Rev Anti Infect Ther. 2007;5:177-184.
4. Fielding J. History of penicillin. Lancet. 1980;1:43-44.

5. Gots JS. The detection of penicillinases-producing properties of microorganisms. Science. 1945;102:309.

6. Smith JT, Hamilton-miller JM, Knox R. Isoxazolyl penicillins and penicillinase. Nature. 1962;195:1300-1301.

7. Moran GJ, Krishnadasan A, Gorwitz RJ, et al. Methicillin-resistant S. aureus infections among patients in the emergency department. $N$ Engl J Med. 2006;355:666-674.

8. Dauwalder O, Lina G, Durand G, et al. Epidemiology of invasive methicillin-resistant Staphylococcus aureus clones collected in France in 2006 and 2007. J Clin Microbiol. 2008;46:3454-3458.

9. Holmes A, Ganner M, McGuane S, Pitt TL, Cookson BD, Kearns AM. Staphylococcus aureus isolates carrying Panton-Valentine leucocidin genes in England and Wales: frequency, characterization, and association with clinical disease. J Clin Microbiol. 2005;43: 2384-2390.

10. Chini V, Petinaki E, Foka A, Paratiras S, Dimitracopoulos G, Spiliopoulou I. Spread of Staphylococcus aureus clinical isolates carrying Panton-Valentine leukocidin genes during a 3-year period in Greece. Clin Microbiol Infect. 2006;12:29-34.

11. Ramdani-Bouguessa N, Bes M, Meugnier H, et al. Detection of methicillin-resistant Staphylococcus aureus strains resistant to multiple antibiotics and carrying the Panton-Valentine leukocidin genes in an Algiers hospital. Antimicrob Agents Chemother. 2006;50: 1083-1085.

12. Centers of disease control and prevention national nosocomial infections surveillance system: MRSA among ICU patients, 1995-2004.

13. Micek ST. Alternatives to vancomycin for the treatment of methicillinresistant Staphylococcus aureus infections. Clin Infect Dis. 2007;45 Suppl 3:S184-S190.

14. Sivagnanam S, Deleu D. Red man syndrome. Crit Care. 2003;7: 119-120.

15. Von Drygalski DA, Curtis BR, Bougie DW, et al. Vancomycin-induced immune thrombocytopenia. $N$ Engl J Med. 2007;356:904-910.

16. Blot S. MRSA pneumonia: better outcome through continuous infusion of vancomycin? Crit Care Med. 2005;33:2127-2128.

17. Byl B, Jacobs F, Wallemacq P, et al. Vancomycin penetration of uninfected pleural fluid exudate after continuous or intermittent infusion. Antimicrob Agents Chemother. 2003;47:2015-2017.

18. Di FA, De Gaudio AR, Novelli A, et al. Continuous infusion of vancomycin in methicillin-resistant staphylococcus infection. Chemotherapy. 1998;44:63-68.

19. Steinkraus G, White R, Friedrich L. Vancomycin MIC creep in non-vancomycin-intermediate Staphylococcus aureus (VISA), vancomycin-susceptible clinical methicillin-resistant $\mathrm{S}$. aureus (MRSA) blood isolates from 2001-05. J Antimicrob Chemother. 2007.

20. Tenover FC, Lancaster MV, Hill BC, et al. Characterization of staphylococci with reduced susceptibilities to vancomycin and other glycopeptides. J Clin Microbiol. 1998;36:1020-1027.

21. Kirst HA, Thompson DG, Nicas TI. Historical yearly usage of vancomycin. Antimicrob Agents Chemother. 1998;42:1303-1304.

22. Silverman JA, Oliver N, Andrew T, Li T. Resistance studies with daptomycin. Antimicrob Agents Chemother. 2001;45:1799-1802.

23. Wiedemann B. Test results: characterising the antimicronial activity of daptomycin. Clin Microbiol Infect. 2006;12:9-14.

24. Rose WE. LMRJ: In vitro activity of daptomycin in combination with gentamycin and rifampicin against Staphylococcus aureus isolates with reduced susceptibility from the bacterial endocarditis trial [abstract]. Interscience Conference on Antimicrobial Agents and Chemotherapy. September 17-20, 2007; Chicago, Illinois: A-794.

25. Tally FP, DeBruin MF. Development of daptomycin for gram-positive infections. J Antimicrob Chemother. 2000;46:523-526.

26. Kirst HA, Thompson DG, Nicas TI. Historical yearly usage of vancomycin. Antimicrob Agents Chemother. 1998;42:1303-1304. 
27. Cubist Pharmaceuticals. Cubicin full prescribing information. 2006.

28. Tedesco KL, Rybak MJ. Daptomycin. Pharmacotherapy. 2004; 24:41-57.

29. Lakey JH, Ptak M. Fluorescence indicates a calcium-dependent interaction between the lipopeptide antibiotic LY146032 and phospholipid membranes. Biochemistry. 1988;27:4639-4645.

30. Silverman JA, Perlmutter NG, Shapiro HM. Correlation of daptomycin bactericidal activity and membrane depolarization in Staphylococcus aureus. Antimicrob Agents Chemother. 2003;47:2538-2544.

31. Dvorchik BH, Brazier D, DeBruin MF, Arbeit RD. Daptomycin pharmacokinetics and safety following administration of escalating doses once daily to healthy subjects. Antimicrob Agents Chemother. 2003;47:1318-1323.

32. Bush LM, Boscia JA, Wendeler M, Pitsakis PG, Kaye D. In vitro postantibiotic effect of daptomycin (LY146032) against Enterococcus faecalis and methicillin-susceptible and methicillin-resistant Staphylococcus aureus strains. Antimicrob Agents Chemother. 1989;33:1198-1200.

33. Hanberger H, Nilsson LE, Maller R, Isaksson B. Pharmacodynamics of daptomycin and vancomycin on Enterococcus faecalis and Staphylococcus aureus demonstrated by studies of initial killing and postantibiotic effect and influence of $\mathrm{Ca}^{+}$ and albumin on these drugs. Antimicrob Agents Chemother. 1991;35:1710-1716.

34. Wise R, Gee T, Andrews JM, Dvorchik B, Marshall G. Pharmacokinetics and inflammatory fluid penetration of intravenous daptomycin in volunteers. Antimicrob Agents Chemother. 2002;46:31-33.

35. Pryka RD, Novak RM, Wagner DK, Rodvold KA. Clinical pharmacokinetics of daptomycin. DICP. 1990;24:255-256.

36. Trotman RL, Williamson JC, Shoemaker DM, Salzer WL. Antibiotic dosing in critically ill adult patients receiving continuous renal replacement therapy. Clin Infect Dis. 2005;41:1159-1166.

37. Oleson FB Jr, Berman CL, Kirkpatrick JB, Regan KS, Lai JJ, Tally FP. Once-daily dosing in dogs optimizes daptomycin safety. Antimicrob Agents Chemother. 2000;44:2948-2953.

38. Arbeit RD, Maki D, Tally FP, Campanaro E, Eisenstein BI. The safety and efficacy of daptomycin for the treatment of complicated skin and skin-structure infections. Clin Infect Dis. 2004;38:1673-1681.

39. Figueroa DA. MEA-GVBFCWWUCS-MS: Clinical safety with daptomycin at doses greater than $6 \mathrm{mg} / \mathrm{kg}$ [abstract]. Interscience Conference on Antimicrobial Agents and Chemotherapy. September 17-20, 2007; Chicago, Illinois: A-792.

40. Patel SJ, Samo TC, Suki WN. Early-onset rhabdomyolysis related to daptomycin use. Int J Antimicrob Agents. 2007;30:472-474.

41. Fowler VG Jr, Boucher HW, Corey GR, et al. Daptomycin versus standard therapy for bacteremia and endocarditis caused by Staphylococcus aureus. $N$ Engl J Med. 2006;355:653-665.

42. Weis F, Beiras-Fernandez A, Schelling G. Daptomycin, a lipopeptide antibiotic in current clinical practice. Curr Opin Investig Drugs. 2008;9:879-884.

43. Arbeit RD, Maki D, Tally FP, Campanaro E, Eisenstein BI. The safety and efficacy of daptomycin for the treatment of complicated skin and skin-structure infections. Clin Infect Dis. 2004;38:1673-1681.

44. Owens RC Jr, Lamp KC, Friedrich LV, Russo R. Postmarketing clinical experience in patients with skin and skin-structure infections treated with daptomycin. Am J Med. 2007;120:S6-S12.

45. Fowler VG Jr, Boucher HW, Corey GR, et al. Daptomycin versus standard therapy for bacteremia and endocarditis caused by Staphylococcus aureus. N Engl J Med. 2006;355:653-665.

46. Falagas ME, Giannopoulou KP, Ntziora F, Papagelopoulos PJ. Daptomycin for treatment of patients with bone and joint infections: a systematic review of the clinical evidence. Int J Antimicrob Agents. 2007;30:202-209.
47. Antony SJ. Combination therapy with daptomycin, vancomycin, and rifampin for recurrent, severe bone and prosthetic joint infections involving methicillin-resistant Staphylococcus aureus. Scand J Infect Dis. 2006;38:293-295.

48. Rao N, Regalla DM. Uncertain efficacy of daptomycin for prosthetic joint infections: a prospective case series. Clin Orthop Relat Res. 2006;451:34-37.

49. Finney MS, Crank CW, Segreti J. Use of daptomycin to treat drug-resistant Gram-positive bone and joint infections. Curr Med Res Opin. 2005;21:1923-1926.

50. Falagas ME, Giannopoulou KP, Ntziora F, Papagelopoulos PJ. Daptomycin for treatment of patients with bone and joint infections: a systematic review of the clinical evidence. Int $J$ Antimicrob Agents. 2007;30:202-209.

51. Eifert S, Kronschnabl S, Kaczmarek I, Reichart B, Vicol C. Omental flap for recurrent deep sternal wound infection and mediastinitis after cardiac surgery. Thorac Cardiovasc Surg. 2007;55:371-374.

52. Lamp KC, Friedrich LV, Mendez-Vigo L, Russo R. Clinical experience with daptomycin for the treatment of patients with osteomyelitis. Am J Med. 2007;120:S13-S20.

53. Carlyn CJ, Baltch AL, George M, Smith RP. Daptomycin in the treatment of persistent bacteremia with invasive complications caused bay a small colony variant of methicillin-resistant Staphylococcus aureus in an orthopedic patient. Infect Dis Clin Pract. 2006;14: 394-398.

54. Cunha BA, Eisenstein LE, Hamid NS. Pacemaker-induced Staphylococcus aureus mitral valve acute bacterial endocarditis complicated by persistent bacteremia from a coronary stent: Cure with prolonged/high-dose daptomycin without toxicity. Heart Lung. 2006;35:207-211.

55. Cunha BA, Hamid N, Kessler H, Parchuri S. Daptomycin cure after cefazolin treatment failure of Methicillin-sensitive Staphylococcus aureus (MSSA) tricuspid valve acute bacterial endocarditis from a peripherally inserted central catheter (PICC) line. Heart Lung. 2005; 34 : $442-447$.

56. Weis F, Beiras-Fernandez A, Kaczmarek I, et al. Daptomycin for eradication of a systemic infection with a methicillin-resistantStaphylococcus aureus in a biventricular assist device recipient. Ann Thorac Surg. 2007;84:269-270.

57. Presterl E. KCRSLWGW: Activity of daptomycin and azithromycin against established biofilms formed by Staphylococcus epidermidis isolates in cardiac device infections [abstract]. Interscience Conference on Antimicrobial Agents and Chemotherapy. September 17-20, 2007; Chicago, Illinois: K-1117.

58. Wagenlehner FM, Lehn N, Witte W, Naber KG. In vitro activity of daptomycin versus linezolid and vancomycin against gram-positive uropathogens and ampicillin against enterococci, causing complicated urinary tract infections. Chemotherapy. 2005;51:64-69.

59. Enoch DA, Bygott JM, Daly ML, Karas JA. Daptomycin. J Infect. 2007;55:205-213.

60. Silverman JA, Mortin LI, Vanpraagh AD, Li T, Alder J. Inhibition of daptomycin by pulmonary surfactant: in vitro modeling and clinical impact. J Infect Dis. 2005;191:2149-2152.

61. Voorn GP, Kuyvenhoven J, Goessens WH, et al. Role of tolerance in treatment and prophylaxis of experimental Staphylococcus aureus endocarditis with vancomycin, teicoplanin, and daptomycin. Antimicrob Agents Chemother. 1994;38:487-493.

62. Sakoulas G, Eliopoulos GM, Alder J, Eliopoulos CT. Efficacy of daptomycin in experimental endocarditis due to methicillin-resistant Staphylococcus aureus. Antimicrob Agents Chemother. 2003; 47:1714-1718.

63. Caron F, Kitzis MD, Gutmann L, et al. Daptomycin or teicoplanin in combination with gentamicin for treatment of experimental endocarditis due to a highly glycopeptide-resistant isolate of Enterococcus faecium. Antimicrob Agents Chemother. 1992;36:2611-2616. 
64. Ramos MC, Grayson ML, Eliopoulos GM, Bayer AS. Comparison of daptomycin, vancomycin, and ampicillin-gentamicin for treatment of experimental endocarditis caused by penicillin-resistant enterococci. Antimicrob Agents Chemother. 1992;36:1864-1869.

65. Cunha BA, Eisenstein LE, Hamid NS. Pacemaker-induced Staphylococcus aureus mitral valve acute bacterial endocarditis complicated by persistent bacteremia from a coronary stent: Cure with prolonged/high-dose daptomycin without toxicity. Heart Lung. 2006;35:207-211.

66. Falagas ME, Giannopoulou KP, Ntziora F, Vardakas KZ. Daptomycin for endocarditis and/or bacteraemia: a systematic review of the experimental and clinical evidence. J Antimicrob Chemother. 2007;60: 7-19.
67. Muangsiri W, Kirsch LE. The protein-binding and drug release properties of macromolecular conjugates containing daptomycin and dextran. Int J Pharm. 2006;315:30-43.

68. Noel SP, Courtney H, Bumgardner JD, Haggard WO. Chitosan films: a potential local drug delivery system for antibiotics. Clin Orthop Relat Res. 2008;466:1377-1382.

\section{Publish your work in this journal}

Infection and Drug Resistance is an international, peer-reviewed openaccess journal that focuses on the optimal treatment of infection (bacterial, fungal and viral) and the development and institution of preventive strategies to minimize the development and spread of resistance. The journal is specifically concerned with the epidemiology of antibiotic

\section{Dovepress}

resistance and the mechanisms of resistance development and diffusion in both hospitals and the community. The manuscript management system is completely online and includes a very quick and fair peerreview system, which is all easy to use. Visit http://www.dovepress.com/ testimonials.php to read real quotes from published authors.

Submit your manuscript here: http://www.dovepress.com/infection-and-drug-resistance-journal 